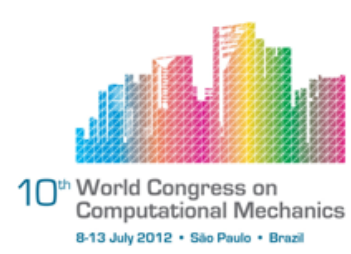

\title{
VIBRATION OF SLENDER STRUCTURE WITH LARGE DEFORMATION, CONTACT AND FLOW
}

\author{
N. Al Sayed ${ }^{1}$ and E. Longatte ${ }^{1}$ \\ ${ }^{1}$ LaMSID- UMR 8193 CNRS-EDF-CEA, 1 Avenue du Général de Gaulle, 92140 Cla- \\ mart, France (nazir-externe.al-sayed@edf.fr)
}

\begin{abstract}
The main purpose of this work is to simulate the displacement in a fluid medium, of a rigid cylinder contained in another fixed one. This phenomena is called annular cavity. To fulfill our goal, we used the Arbitrary Lagrangian-Eulerian method, to describe the fluid-structure interface and treat the mesh's deformation. Finally, a numerical test is performed, on one hand to study the numerical stability, and on the other hand to determine the contact force between the two cylinders.
\end{abstract}

Keywords: Fluid-Structure Interaction, Annular Cavity, Stability, Contact analysis.

\section{INTRODUCTION}

The aim of this work is to study deformations of a rigid structure with respect to the mechanical stress induced by a fluid in a confined area. Indeed, the main problem of this phenomena is how to represent the forces of interaction by using the Eulerian formulation for the fluid and the Lagrangian formulation for the structure [7]. There are two approaches to simulate this phenomena: Fixed mesh methods [9, 15, 10, 5] and the Arbitrary Lagrangian-Eulerian method (ALE) which is used in the present work Code_Saturne [11, 3].

The outline of this paper is the following: In section 2, we will be describing the problem of fluid-structure interaction. In section 3, we explain the concept of the Arbitrary Lagrangian-Eulerian method, in order to represent the fluid-structure interface. Finally, by the use of Code_Saturne, a numerical test contribute on one hand, to study the structure's displacement and the numerical stability, and on the other hand to determine the contact force between both cylinders.

\section{FORMULATION OF FLUID AND STRUCTURE PROBLEMS}

One consider a fluid domain $\Omega_{f}$ in an incompressible, Newtonian, isothermal context, and a solid one $\Omega_{s}$. One denote $\Gamma(t)$ the interface between the unsteady fluid 
domain $\Omega_{f}(t)$ and the solid domain $\Omega_{s}(t)$. Also, one define the domain $\Omega$ considered to be fixed during time by:

$$
\bar{\Omega}_{f}(t) \bigcup \bar{\Omega}_{s}(t)=\bar{\Omega}(t), \Gamma=\bar{\Omega}_{s}(t) \bigcap \bar{\Omega}_{f}(t), \Omega_{f}(t) \bigcap \Omega_{s}(t)=\emptyset
$$

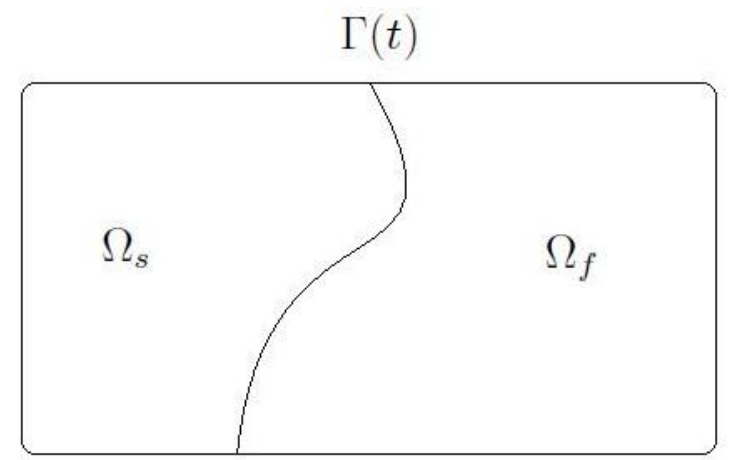

Figure 1. Field of fluid structure interaction

\subsection{Fluid property}

One considers a Newtonian viscous fluid, in an incompressible, isothermal, homogeneous and isotropic framework, it is then described by the conservation of momentum and the continuity equations [14]:

$$
\left\{\begin{array}{l}
\frac{\partial\left(\rho_{f} u\right)}{\partial t}+\operatorname{div}\left(\rho_{f} u \otimes u\right)+\nabla p-2 \mu_{f} \operatorname{div}(D(u))=\rho_{f} f_{e x t} \\
\operatorname{div}(u)=0
\end{array}\right.
$$

where $u=u_{f}$ is the fluid field, $p=p_{f}$ pressure field, $\rho_{f}$ fluid density, $\mu_{f}$ the dynamic viscosity, $f_{\text {ext }}$ the external force density and $D\left(u_{f}\right)$ the strain rate tensor:

$$
D\left(u_{f}\right)=\frac{1}{2}\left[\nabla u+(\nabla u)^{T}\right]
$$

\subsection{Solid property}

One can take the general case of a solid with small deformations, for which the only body force is the gravity one $g$. The motion of the solid domain is described by the conservation of momentum equation [13] as follows:

$$
\rho_{s} \frac{\partial^{2} v}{\partial t^{2}}+\rho_{s} g e_{g}-\nabla \cdot \sigma_{s}=0
$$

where $v$ the displacement of the structure, $\rho_{s}$ is its density, $e_{g}$ the an upward vertical unit vector, $\sigma_{s}$ the solid strem, $\varepsilon$ the deformation of the structure, $\mathrm{E}$ the Young's 
modulus and $\nu_{P}$ the Poisson's ratio. The law describing the elastic behavior of the structure's deformation is the following:

$$
\varepsilon=\frac{1+\nu_{p}}{\mathrm{E}} \sigma_{s}-\frac{\nu_{P}}{\mathrm{E}} \operatorname{tr}\left(\sigma_{s}\right) I_{d}
$$

After choosing a specific model for each of the fluid and the structure, it is necessary to couple these two areas. So, we are facing a problem of fluid-strusture interaction. Before treating this problem numerically, one show first, the necessary conditions for coupling these two areas in order to preserve the equilibrium at the interface.

\subsection{Fluid-structure interface}

- The first condition is to ensure the continuity of velocities at the interface:

$$
\left.u_{f}\right|_{\Gamma}=\left.\dot{v}_{s}\right|_{\Gamma}
$$

- The second condition describes the action-reaction principle. It ensures the continuity of these efforts at the interface $\Gamma$ : This can be mathematically expressed by:

$$
\left.\sigma_{f} \cdot \mathrm{n}_{f}\right|_{\Gamma}=\left.\sigma_{s} \cdot \mathrm{n}_{s}\right|_{\Gamma}
$$

\section{ARBITRARY LAGRANGIAN-EULERIAN method}

\subsection{Fluid formulation}

The ALE method consists in defining an arbitrary domain $\Omega_{a}$ which correspond to an arbitrary discretization of the domain $\Omega_{t}[7]$, whose nodes on the fluid domain boundaries coincide with the Eulerian mesh ones. In $\Omega_{a}$, a point $M$ is identified by the independent variables $\xi$ and a continuous function $\Psi$ as follows:

$$
x=\Psi(\xi, t)
$$

The Lagrangian velocity at the point $M$ is identified as follows:

$$
w(t, x)=\frac{\partial \Psi(\xi, t)}{\partial t} .
$$

Using this notation for all dependent variables $f$ in the Eulerian domain one gets:

$$
f(t, x)=f(\varphi(t, x), t) .
$$

As for its Lagrangian (or material) derivative, we also write:

$$
\frac{d f}{d t}=\frac{\partial f}{\partial t}+u . \nabla_{x} f
$$

We also note that: 


$$
f(t, x)=f(\Psi(\xi, t), t)=\widehat{f}(\xi, t) .
$$

Then, the Lagrangian derivative is:

$$
\frac{\partial \widehat{f}}{\partial t}=\frac{\partial f}{\partial t}+w \cdot \nabla_{x} f .
$$

Finally, using relations (8) et (9), we can write the Lagrangian derivative on the arbitrary domain $\Omega_{a}$ :

$$
\frac{d f}{d t}=\frac{\partial \widehat{f}}{\partial t}+(u-w) \cdot \nabla_{x} f
$$

Applying the relation (10) to the velocity field, equations (1) become:

$$
\left\{\begin{array}{l}
\frac{\partial \widehat{u}}{\partial t}+(u-w) \cdot \nabla u+\frac{1}{\rho_{f}} \nabla p-2 \nu_{f} \operatorname{div}(D(u))=f_{\text {ext }}, \\
\operatorname{div}(u)=0
\end{array}\right.
$$

A method for calculating the velocity of the mesh $w$ was proposed by Souli and Zolesio [16]. This method consists in solving a diffusion equation of the form:

$$
\begin{cases}\operatorname{div}(\lambda(\nabla w))=0 & \text { in } \Omega \\ w=\dot{v}_{\text {imposed }} & \text { in } \Gamma .\end{cases}
$$

$\lambda$ is the diffusion coefficient of the mesh. It can control the mesh deformation. Indeed, according to this constant, the mesh becomes distorted, especially near the moving interface $\Gamma$. So, it's necessary to increase its value around the moving interface, and to decrease it in the rest of the domain in order to calculate the efforts.

\subsection{Solid computation}

The discretization of equation (2) by using the finite element method, leads us to solve the following equation:

$$
M_{s} \ddot{v}+C_{s} \dot{v}+K_{s} v+F=0
$$

with $M_{s}$ the solid mass, $C_{s}$ the damping coefficient, $K_{s}$ the stiffness and $F$ the force applied on the structure.

The algorithm adopted is that of Newmark, which is defined as follows:

$$
\begin{gathered}
M_{s} \ddot{v}^{n+1}+C_{s} \dot{v}^{n+1}+K_{s} v^{n+1}+F^{n+1}=0, \\
\dot{v}^{n+1}=\dot{v}^{n}+\Delta t\left[(1-\alpha) \ddot{v}^{n}+\alpha \ddot{v}^{n+1}\right], \\
v^{n+1}=v^{n}+\Delta t \dot{v}^{n}+\Delta t^{2}\left[\left(\frac{1}{2}-\beta\right) \ddot{v}^{n}+\beta \ddot{v}^{n+1}\right],
\end{gathered}
$$




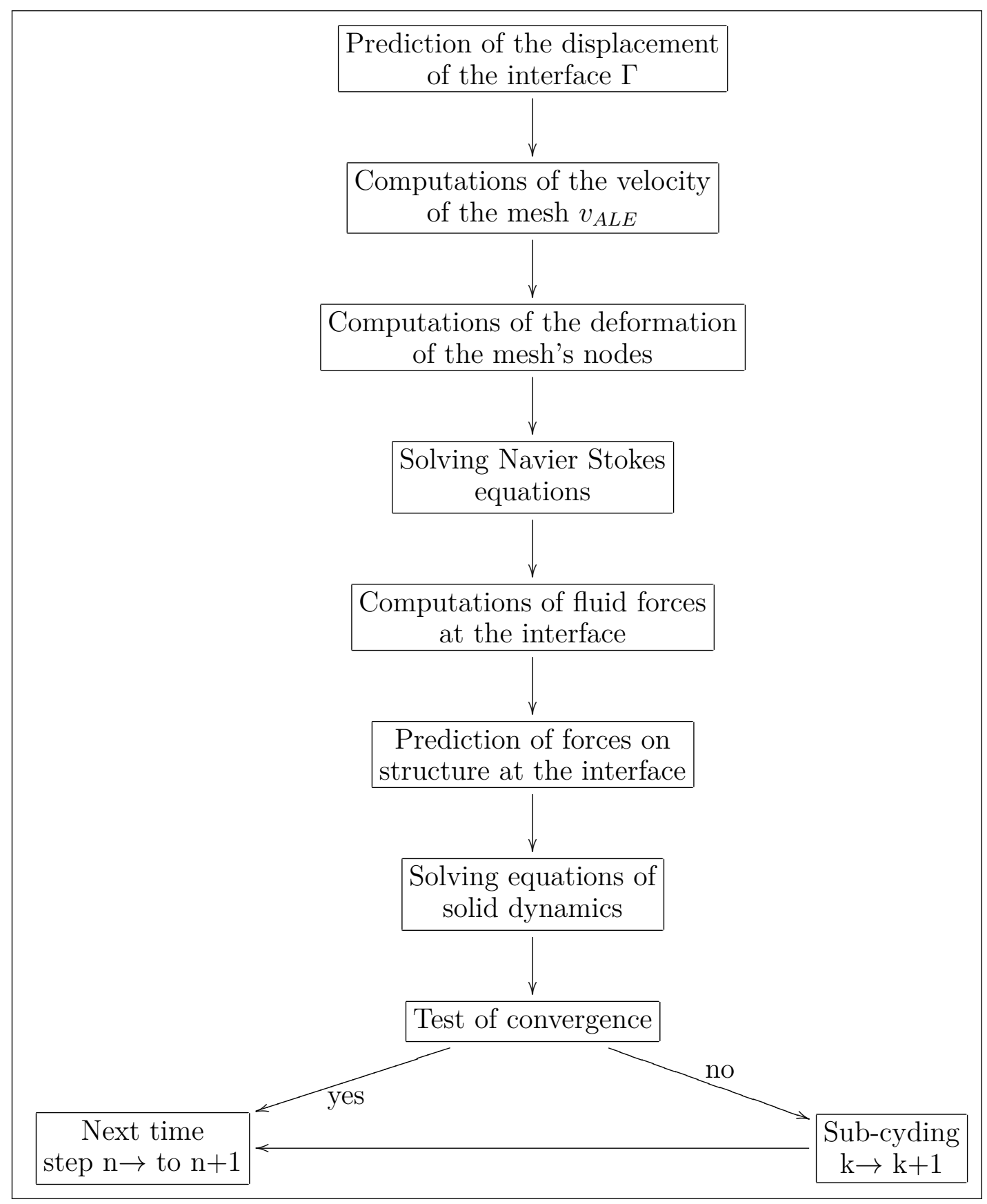

Figure 2. Algorithm 
where $\alpha$ and $\beta$ are two constants.

One introduces relations (15) and (16) in equation (14), to obtain then the value of $\ddot{v}^{n+1}$. Also, from relations (15) and (16), one calculates the values of $\dot{v}^{n+1}$ and $v^{n+1}$.

For $\alpha=\frac{1}{2}$ and $\beta=\frac{1}{4}$, in order to have a scheme of order 2 which is unconditionally stable. The algorithm is summarized in figure 2 .

\section{NUMERICAL VALIDATION}

One considers a fluid between two coaxial cylinders of radius $R_{1}=0.1 \mathrm{~m}$ and $R_{2}=0.2 \mathrm{~m}$. The central cylinder of radius $R_{1}$ is connected to a spring of mass $m_{s}=1 \mathrm{~kg}$, and of stiffness $K_{S}$, while the second one is fixed. One discards the cylinder within a distance $x_{0}=0005 m$ from its equilibrium position and release it without initial velocity, figure 3.

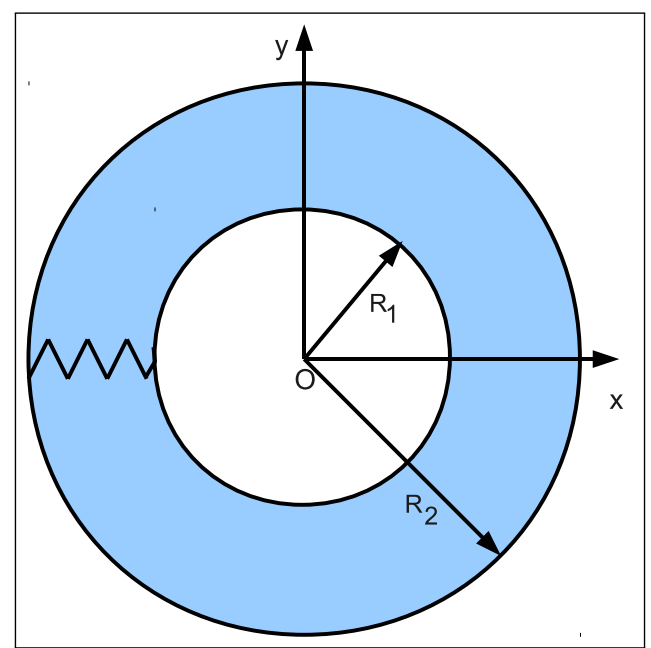

Annular cavity

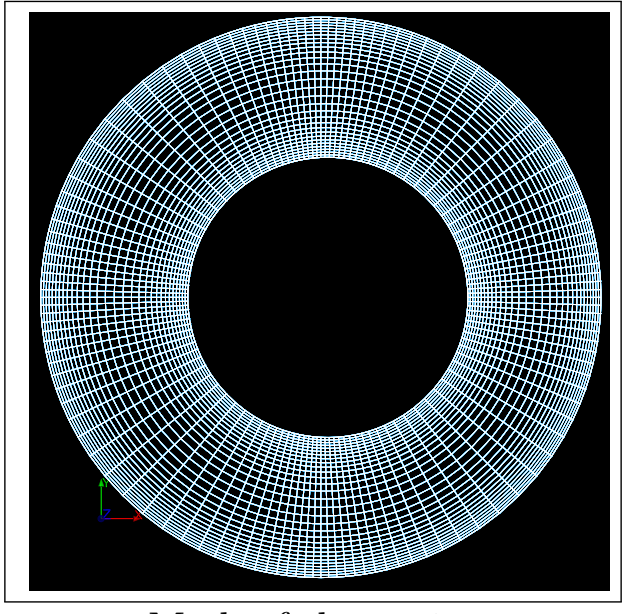

Mesh of the cavity

Figure 3. Geometry of the annular cavity and its quadratic mesh

For fluid computations, the schemes used for the space variable, is the second order centered scheme, as for the time variable we use Crank-Nickolson with a constant time step. The mesh used for this study contains 3600 celles. The inner cylinder is reduced to a single degree of freedom system node in the center mass of gravity. The numerical datas are summarized in table 1:

\begin{tabular}{cccc}
\hline \hline Fluid & Mass & $\Delta t$ & Viscosity \\
\hline Air & $\rho_{\text {air }}=1 \mathrm{kgm}^{-3}$ & $0.0628 \mathrm{~s}$ & $\mu_{\text {air }}=19.610^{-6} \mathrm{kgm}^{-1} \mathrm{~s}^{-1}$ \\
& & & \\
Water & $\rho_{\text {water }}=1000 \mathrm{kgm}^{-3}$ & $0.0628 \mathrm{~s}$ & $\mu_{\text {water }}=0.001 \mathrm{kgm}^{-1} \mathrm{~s}^{-1}$ \\
\hline \hline
\end{tabular}

Table 1. Calculus parameters

The cylinder oscillates around its initial position as shown in figures 4 et 5 . We note that, if there is an air flow without damping, the structure's displacement will be 


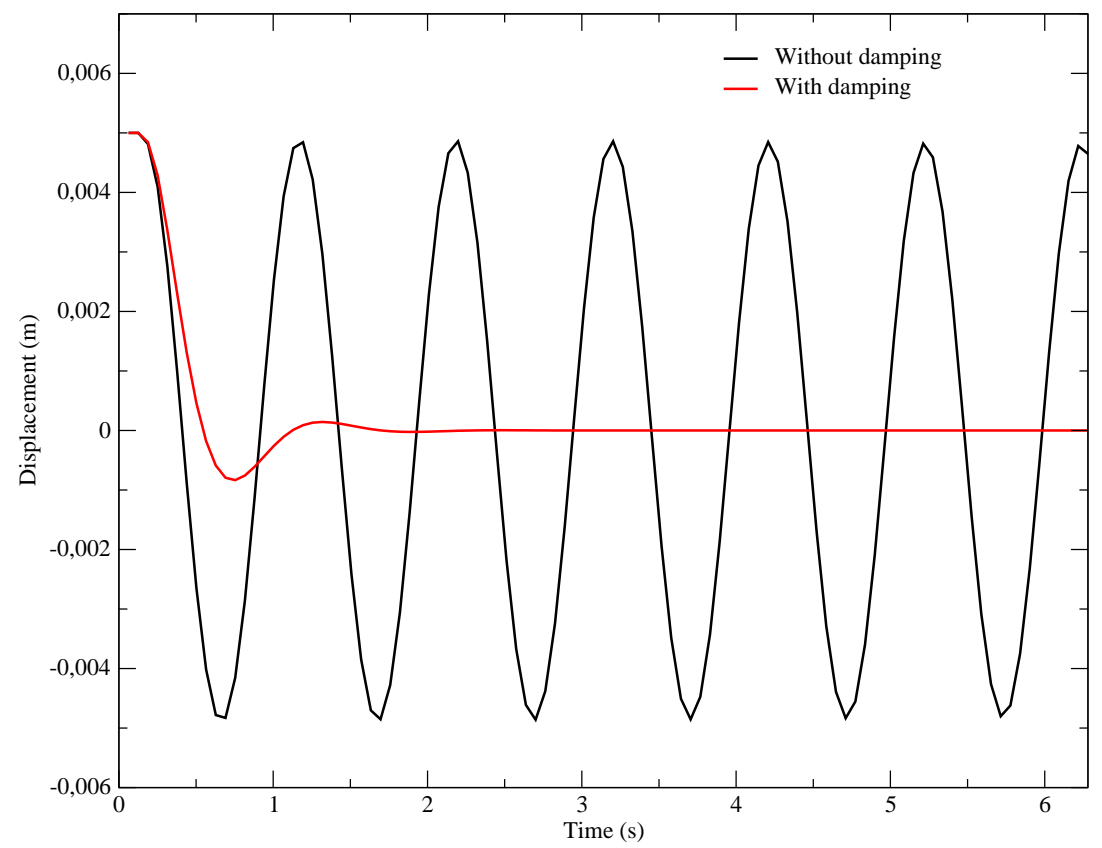

Figure 4. Solid displacement in air for six oscillations: The damping constent, $C_{s}=2 \pi$.

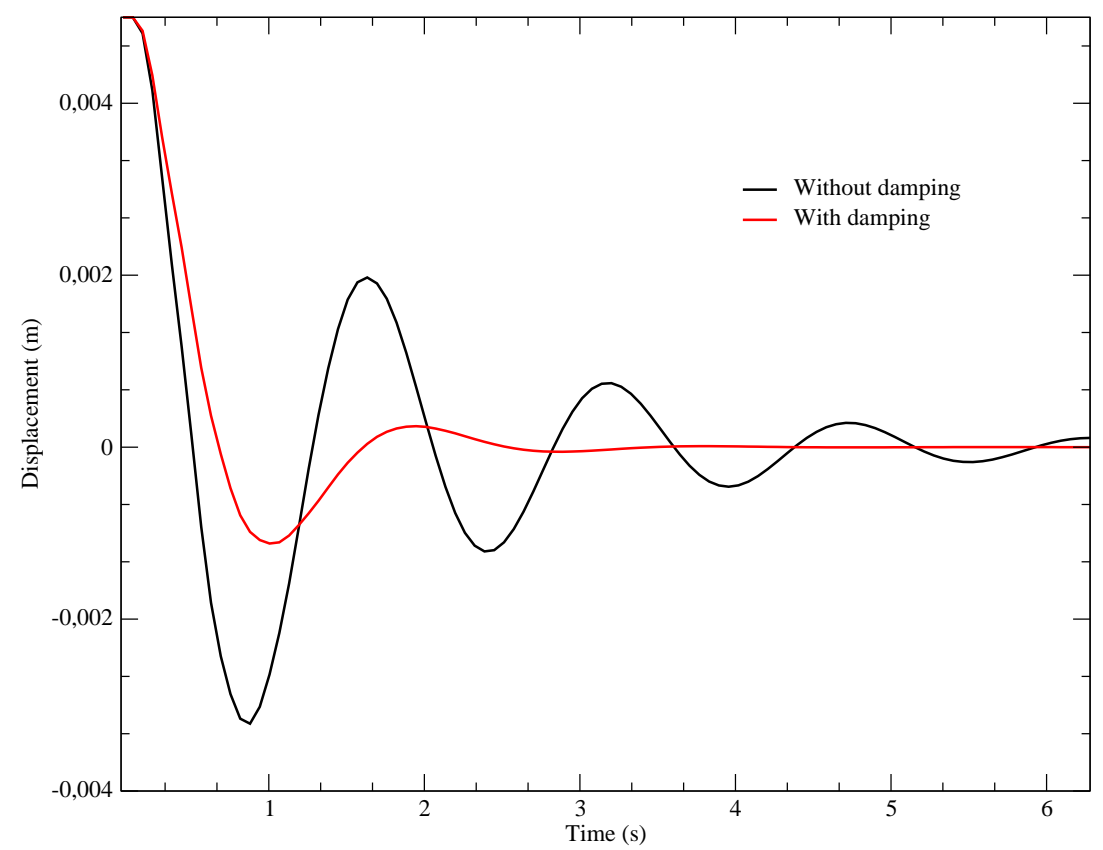

Figure 5. Solid displacement in water for six oscillations: The damping constent, $C_{s}=$ $2 \pi$. 
periodic of period 1, otherwise the cylinder stops after six oscillations because of the friction caused by the water. In the next section, we study the stability depending on the mass and the frequency of the structure.

\subsection{Influence of solid mass on numerical stability}

In this section, we study the influence of changing the mass $m_{s}$ of the structure, on the stability of the flow [14], while keeping the frequency $f=\frac{1}{2 \pi} \sqrt{\left(\frac{k}{m_{s}}\right)}$ constant. M.A. Fernández and al. [8] have developed a criteria to measure the numerical scheme stability. It is defined as follows:

$$
\rho_{s} \geq \frac{C}{H^{\alpha}}\left(\rho_{f} h+2 \frac{\mu_{f} \Delta t}{h}\right),
$$

where $C$ is a constant which does not depend, neither on the flow physics, nor on the mesh size. $\mathrm{H}$ and $\mathrm{h}$ are respectively, the mesh sizes for both structure and fluid, and $\alpha$ is a function defined as follows:

$$
\alpha=\left\{\begin{array}{lll}
0 & \text { si } & \bar{\Omega}_{s}=\Gamma \\
1 & \text { si } & \bar{\Omega}_{s} \neq \Gamma
\end{array}\right.
$$

\begin{tabular}{ccccc}
\hline \hline$\rho_{s}=m_{s}$ & $k$ & $\Delta t$ & Result & Stability condition $(17)$ \\
\hline 1 & $(2 \pi)^{2}$ & $6.2810^{-2}$ & Stable & Verified \\
0.8 & $m_{s}(2 \pi)^{2}$ & $6.2810^{-2}$ & Stable & Verified \\
0.7 & $m_{s}(2 \pi)^{2}$ & $6.2810^{-2}$ & Stable & Verified \\
0.65 & $m_{s}(2 \pi)^{2}$ & $6.2810^{-2}$ & Unstable & Broken \\
0.6 & $m_{s}(2 \pi)^{2}$ & $6.2810^{-2}$ & Unstable & Broken \\
0.5 & $m_{s}(2 \pi)^{2}$ & $6.2810^{-2}$ & Unstable & Broken \\
0.1 & $m_{s}(2 \pi)^{2}$ & $6.2810^{-2}$ & Unstable & Broken \\
\hline \hline
\end{tabular}

Table 2. Numerical stability for several solid mass

In the table 2, we show the test cases we have made. We notice that for a given structure of mass greater or equal than $0.7 \mathrm{~kg}$, the calculations converged. Using these test cases, we compute the value of the constant $C$ which appear in the inequality of M.A. Fernández and al. [8] as the minimum value between all those that ensure convergence of the structure's displacement:

$$
C=\operatorname{Min}\left\{\frac{m_{s}}{\left(\rho_{f} h+2 \frac{\mu_{f} \Delta t}{h}\right)}\right\}=0.62
$$

and $h=\mathcal{O}\left(10^{-3}\right)$.

Otherwise, in the case where the mass of the structure is strictly less than $0.7 \mathrm{~kg}$, the displacement of the structure diverges. This instability is expected and it's consistent with the inequality of M.A. Fernández and al. (17), because $\rho_{s}<\frac{C}{H^{\alpha}}\left(\rho_{f} h+2 \frac{\mu_{f} \Delta t}{h}\right)$. 
In the next section, we compute the contact force between a master surface (fixed wall) and a slave surface (moving wall) by using the penalty method.

\subsection{Contact force}

Before calculating the contact force between two structures, one must locate the contact place, the master surface and the slave one. In general, ain't no method exist for choosing these two surfaces. However, some authors give rules to define these two surfaces $[2,12]$. In theory, there are two methods to calculate the impact force: The Lagrange multipliers method and the penalty one [4].

The Lagrange multipliers method is more expansive in terms of time cost, than the penalization one. For this reason, we will adopt the penalty method:

$$
F=-k_{c h o c} d,
$$

where $k_{c h o c}$ is the stiffness of the shock and $d$ is the distance of penetration.

It's difficult to have a sharp estimate for the value of the shock's stifness. If this value is too low, the interpenetration becomes unacceptable and the fluid passes through the structure. So, to limit penetrations, the stiffness must be high. In $3 D$ there is a formula for calculating the stiffness $[1,6]$ :

$$
k_{c h o c}=r_{f a c} \frac{G S^{2}}{V}
$$

where $G=\frac{E}{2\left(1+\nu_{p}\right)}, S$, and $V$ are respectively, the shear modulus of materials, the contact surface of the master element and its contact volume. $r_{f a c}$ is a scalar factor. The figure 6 shows the result obtained after calculating a contact force of an air flow without friction. We note also that the minimal distance of contact between the master surface and the slave one is equal to $0.104 \mathrm{~m}$.

\section{CONCLUSION}

This work investigates the issue involving in the same time fluid structure interaction and contact between solids. The purpose is to check whether or not an explicit numerical integration of contact forces does not affect the numerical stability of an iterative procedure involved for a fluid solid coupled computation. The example of the annular cavity is used to illustrate the validity limit of the approach.

\section{References}

[1] EUROPLEXUS, A Computer Program for the Finite Element Simulation of Fluid-Structure Systems under Transient Dynamic Loading. EUROPEAN COMMISSION, 2012.

[2] M. Abbas. Formulation discrète du contact-frottement. Documentation de référence de Code Aster [R5.03.50]. 


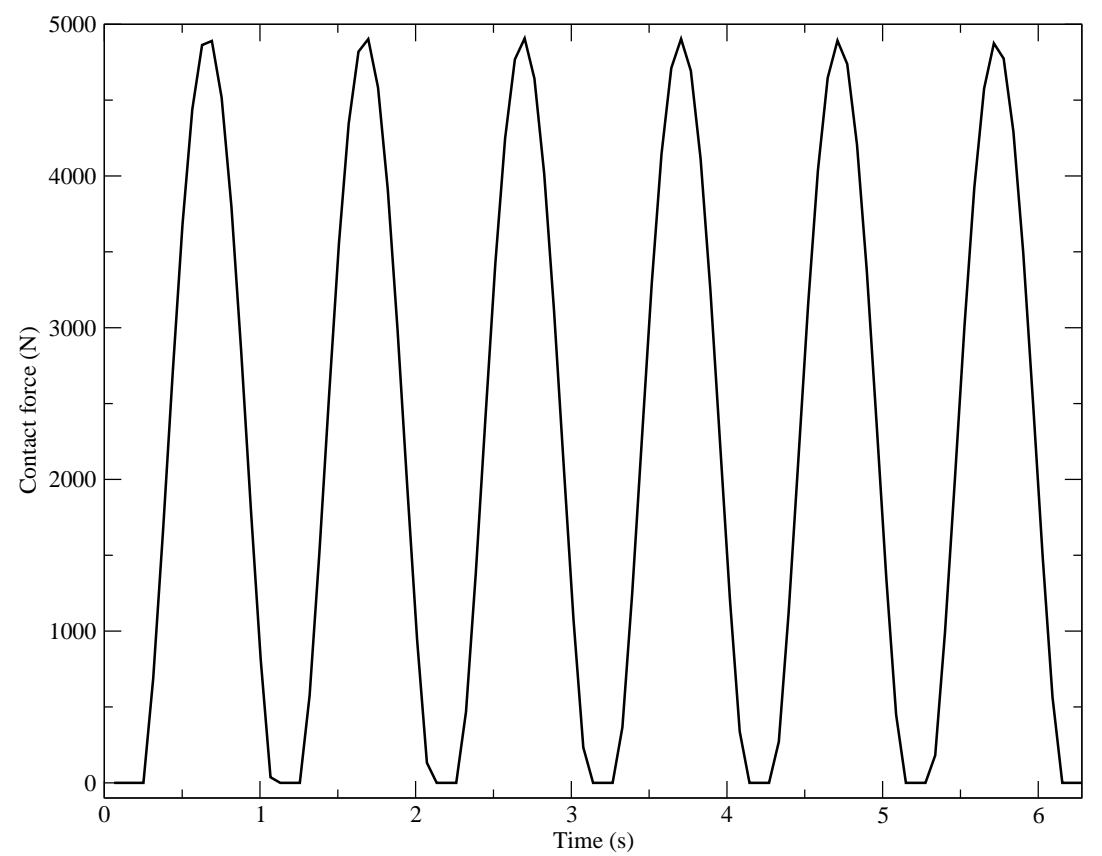

Figure 6. Contact force, with $E=150 M P a, \nu_{p}=0.3, r_{f a c}=1$ and $j e u=0.104 m$

[3] F. Archambeau, N. Méchitoua, and M. Sakiz. Code_Saturne: A finite volume code for the computation of turbulent incompressible flows-industrial applications. Int. J. on Finite Volumes, Electronical edition: http://averoes.math.univ-paris13.fr/html, ISSN 1634(0655), 2004.

[4] T. Belytschko and M. Neal. Contact-impact by the pinball algorithm with penalty and lagrangian methods. International Journal for Numerical Methods in Engineering, 31:547-572, 1991.

[5] S.P.A. Bordas, T. Rabczuk, N-X Hung, V. P. Nguyen, S. Natarajan, T. Bog, D. M. Quan, and N. V. Hiep. Strain smoothing in FEM and XFEM. Computers and Structures, 88:1419-1443, 2010.

[6] F. Casadei, J.P. Halleux, H. Bung, and M. Lepareux. Some tentative guidelines for the development of the EUROPLEXUS software system. Technical Note N. I.00.146, 2000.

[7] J. Donea, A. Huerta, J.-P. Ponthot, A. Rodriguez-Ferran, E. Stein, R. de Borst, and T.J.R. Hughes. Encyclopedia of Computational Mechanics, Volume 1, Chapter 4:413-437, 2004.

[8] M. A. Fernández, J.-F Gerbeau, and C. Grandmont. A projection algorithm for fluid-structure interaction problems with strong added-mass effect. Comptes Rendus Mathématique, 342:279-284, 2006.

[9] Axel Gerstenberger and Wolfgang A. Wall. An eXtended Finite Element Method/Lagrange multiplier based approach for fluid-structure interaction. Comput. Methods Appl. Mech. Engrg., 197:1699-1714, 2008. 
[10] G.Papadakis. A novel pressure-velocity formulation and solution method for fluidstructure interaction problems. Journal of Computational Physics, 227:3383-3404, 2008 .

[11] C. Hirth, A.A. Amsden, and J. Cook. An arbitrary Lagrangian-Eulerian computing method for all flow speeds. Journal of Computational Physics, 14:227-253, 1974.

[12] A. KONTER. Advanced finite element contact benchmarks. NAFEMS, 2006.

[13] H.J.-P Morand and R. Ohayon. Fluid-Structure Interactions. Wiley, 1995.

[14] M. P. Païdoussis, S. J. Price, and E. de Langre. Fluid-Structure Interactions. Cambridge, 2011.

[15] C. Peskin. The immersed boundary method. Cambridge University Press, Acta Numerica:1-39, 2002.

[16] M. Souli and J. Zolesio. Arbitrary Lagragian-Eulerian and free surface methods in fluid mechanics. Computer Methods in Applied Mechanics and Engineering, 191 (3-5):451-466, 2001. 